\title{
ON TWO PROBLEMS \\ OF ELECTRICAL HEATING OF CONDUCTORS
}

BY

GIOVANNI CIMATTI

Universita di Pisa, Pisa, Italy

\begin{abstract}
The thermal effects of the currents induced in a massive conductor by an external slowly varying magnetic field are studied with regard to existence and uniqueness of solutions. In the first part a theorem of existence of solution is also given for the thermistor problem with a current limiting device.
\end{abstract}

1. Introduction. In this paper we study various problems related to the Joule heating of a conductor. In Sec. 2 a theorem of existence is given for a version of the "thermistor problem" first proposed in [3] and then studied for special boundary conditions in [1]. The boundary conditions here considered are quite general and the same methods apply even to more general situations. In Sec. 3 a model is proposed for describing the Joule heating due to the eddy currents in a very long cylinder when the external variable magnetic field is parallel to the axis of the cylinder. A theorem of existence is given for this problem.

2. The thermistor problem with a current limiting device. Let us consider a solid body conductor of heat and electricity represented by an open, bounded, and connected subset $\Omega$ of $\mathbb{R}^{3}$. The boundary $\partial \Omega \in C^{2}$ consists of three parts, $S_{i}, i=$ $0,1,2$, such that $S_{0} \neq \varnothing, S_{1} \neq \varnothing, S_{i} \cap S_{j}=\varnothing$ when $i \neq j$, and $\partial \Omega=S_{0} \cup S_{1} \cup S_{2}$. $S_{0}$ and $S_{1}$ represent the metallic electrodes to which a difference of potential is applied. $S_{2}$ is insulated both thermally and electrically. The conductor is connected in series with a current limiting device of total resistance $R$. Steady conditions are supposed. $\mathbf{J}$ is the current density, $\mathbf{E}$ the electric field, $\mathbf{q}$ the heat flow, and $u$ the temperature. The electric and thermal conductivities are given functions of the temperature denoted by $\sigma(u)$ and $\kappa(u)$ respectively. From the constitutive relations

$$
\mathbf{J}=\sigma \mathbf{E}, \quad \mathbf{q}=-\kappa \nabla u,
$$

and the usual conservation laws

$$
\nabla \bullet \mathbf{J}=0, \quad \nabla \bullet \mathbf{q}=\mathbf{E} \bullet \mathbf{J},
$$

Received January 23, 1990. 
we obtain, since $\mathbf{E}=-\nabla \varphi$,

$$
\begin{gathered}
\nabla \bullet(\sigma(u) \nabla \varphi)=0, \\
-\nabla \bullet(\kappa(u) \nabla u)=\sigma(u)|\nabla \varphi|^{2} .
\end{gathered}
$$

Denoting by $\mathbf{n}$ the exterior pointing unit normal vector to $\partial \Omega$, we have

$$
i=-\int_{S_{0}} \mathbf{J} \bullet \mathbf{n} d s=\int_{S_{0}} \sigma(u) \frac{d \varphi}{d n} d s
$$

for the total current $i$ entering through $S_{0}$. The surfaces $S_{0}$ and $S_{1}$ are almost exactly equipotential, hence we have

$$
\varphi=0 \quad \text { on } S_{0}, \quad \varphi=\varphi_{1} \text { on } S_{1},
$$

where $\varphi_{1}$ is an unknown constant satisfying the condition

$$
V-R \int_{S_{1}} \sigma(u) \frac{d \varphi}{d n} d s-\varphi_{1}=0,
$$

which follows from $V=\varphi_{1}+R i . V$ is the total difference of applied potential, a given positive constant. Since $S_{2}$ is insulated, we have

$$
\begin{array}{ll}
\frac{d \varphi}{d n}=0 & \text { on } S_{2}, \\
\frac{d u}{d n}=0 & \text { on } S_{2} .
\end{array}
$$

On the remaining part of the boundary we assume for the temperature a Dirichlet boundary condition, i.e.,

$$
u=u_{0} \quad \text { on } S_{0} \cup S_{1} \text {, }
$$

where $u_{0} \geq 0$ is the restriction to $S_{0} \cup S_{1}$ of a $C^{2}(\bar{\Omega})$-function. We arrive to the following problem $\mathrm{Pb}_{1}$ :

Find $\varphi(x)$ and $u(x)$ such that (2.3) and (2.4) hold in $\Omega$. Moreover (2.6), (2.7), (2.8), (2.9), and (2.10) must be satisfied on $\partial \Omega$.

Assume

$$
\begin{gathered}
\sigma(\zeta) \in C^{1}\left(\mathbb{R}_{+}^{1}\right), \quad \kappa(\zeta) \in C^{1}\left(\mathbb{R}_{+}^{1}\right) \\
\sigma_{1} \geq \sigma(\zeta) \geq \sigma_{0}>0 \quad \forall \zeta \geq 0 \\
\kappa(\zeta)>0 \quad \forall \zeta \geq 0 \\
\int_{0}^{\infty} \frac{\kappa(\zeta)}{\sigma(\zeta)} d \zeta=\infty
\end{gathered}
$$

In the sequel we need the following

Lemma 2.1. Given $a(x) \in C^{0}(\bar{\Omega}), b(x) \in C^{0}\left(S_{1}\right)$, and a constant $V>0$ such that

$$
\begin{array}{ll}
a(x) \geq a_{0}>0, & x \in \bar{\Omega}, \\
b(x) \geq b_{0}>0, & x \in S_{1},
\end{array}
$$


the problem

$$
\begin{gathered}
\nabla \bullet(a(x) \nabla \varphi)=0 \quad \text { in } \Omega, \\
\varphi=0 \text { on } S_{0}, \\
\varphi=V-\int_{S_{1}} b \frac{d \varphi}{d n} d s \quad \text { on } S_{1}, \\
\frac{d \varphi}{d n}=0 \quad \text { on } S_{2}
\end{gathered}
$$

has one and only one regular solution.

Proof. Uniqueness. Let $\varphi$ and $\varphi^{\prime}$ be two solutions. Define $\psi=\varphi-\varphi^{\prime}$. The function $\psi$ satisfies

$$
\begin{gathered}
\nabla \bullet(a(x) \nabla \psi)=0 \quad \text { in } \Omega, \\
\psi=0 \text { on } S_{0}, \\
\psi=-\int_{S_{1}} b \frac{d \psi}{d n} d s \quad \text { on } S_{1}, \\
\frac{d \psi}{d n}=0 \text { on } S_{2} .
\end{gathered}
$$

Let $\psi_{1}$ be the constant value of $\psi$ on $S_{1}$. We claim that $\psi_{1} \geq 0$. Assume by contradiction $\psi_{1}<0$. By the maximum principle in Hopf's form [7] we have $\frac{d \psi}{d n}<0$ on $S_{1}$, hence by (2.23) we obtain $\psi_{1}>0$. Similarly we have $\psi_{1} \leq 0$. Therefore $\psi_{1}=0$. This implies $\psi=0$ in $\bar{\Omega}$.

Existence. Let $\Gamma \in[0, V]$ and $\varphi(x ; \Gamma)$ be the unique solution of the problem

$$
\begin{gathered}
\nabla \bullet(a(x) \nabla \varphi)=0 \quad \text { in } \Omega, \\
\varphi=0 \quad \text { on } S_{0}, \quad \varphi=\Gamma \quad \text { on } S_{1}, \quad \frac{d \varphi}{d n}=0 \quad \text { on } S_{2} .
\end{gathered}
$$

By standard results, $\varphi(x ; \Gamma)$ is regular and depends continuously on $\Gamma$. Define

$$
g(\Gamma)=V-\Gamma-\int_{S_{1}} b \frac{d \varphi}{d n} d s
$$

We have $g(\Gamma) \in C^{0}[0, V], g(0)=V$, and $g(V)=-\int_{S_{1}} b \frac{d \varphi}{d n} d s<0$, since $\frac{d \varphi}{d n}>0$ on $S_{1}$. Hence there exists $\phi_{0} \in(0, V)$ such that $g\left(\phi_{0}\right)=0$. The solution of problem $(2.17)-(2.20)$ is given by $\varphi\left(x ; \phi_{0}\right)$.

Lemma 2.2. Let $(\varphi, u)$ be any solution of problem $\mathrm{Pb}_{1}$. We have

$$
\begin{gathered}
0<\varphi<V \quad \text { in } \Omega, \\
\frac{d \varphi}{d n}>0 \quad \text { on } S_{1} .
\end{gathered}
$$

Proof. Let $\varphi_{1}$ be the constant value of $\varphi$ on $S_{1}$. We claim that $\varphi_{1}>0$. By contradiction, let $\varphi_{1}=0$. This implies $\varphi=0$ in $\Omega$ against (2.7) since $V>0$. On the other hand, if $\varphi_{1}<0$ we have, by the maximum principle, $\frac{d \varphi}{d n}<0$ on $S_{1}$ 
which contradicts (2.7). Hence $\varphi_{1}>0$. Therefore $\frac{d \varphi}{d n}>0$ on $S_{1}$ and by (2.7) we have $\varphi_{1}<V$. Again by the maximum principle we conclude that (2.25) and (2.26) hold.

Define $u_{m}=\min _{S_{0} \cup S_{1}} u_{0}$ and $u_{M}=\max _{S_{0} \cup S_{1}} u_{0}$. We introduce now the basic transformation of the problem. Define:

$$
F(u)=\int_{u_{m}}^{u} \frac{\kappa(t)}{\sigma(t)} d t
$$

and

$$
\theta=\varphi^{2} / 2+F(u)
$$

If $(\varphi(x), u(x))$ is a solution of problem $\mathrm{Pb}_{1}$, it is easy to verify that $\theta(x)$ given by (2.28) satisfies

$$
\nabla \bullet(\sigma(u) \nabla \theta)=0 \text { in } \Omega \text {. }
$$

Moreover, evaluating $\theta(x)$ on $\partial \Omega$ we have $\theta=\theta_{0}(x)$ on $S_{0}$ with $\theta_{0}(x)=$ $F\left(u_{0}(x)\right) \geq 0, \theta=\theta_{1}(x)$ on $S_{1}$, where $\theta_{1}(x)=F\left(u_{0}(x)\right)+\varphi_{1}^{2} / 2, x \in S_{1}$, and $\frac{d \theta}{d n}=0$ on $S_{2}$ by (2.8), (2.9). Define

$$
\theta_{M}=V^{2} / 2+F\left(u_{M}\right)
$$

We have

$$
0 \leq \theta \leq \theta_{M} \text { on } S_{0} \cup S_{1}
$$

by $(2.25)$ and, by the maximum principle,

$$
0 \leq \theta \leq \theta_{M} \text { in } \bar{\Omega} \text {. }
$$

Since $\lim _{t \rightarrow \infty} F(t)=\infty$ and $F^{\prime}(t)>0$, we can consider

$$
u=F^{-1}\left(\theta-\varphi^{2} / 2\right) \text {. }
$$

Let us define

$$
\hat{u}=\max _{(\varphi, \theta) \in\left[0, \theta_{M}\right] \times[0, V]} F^{-1}\left(\theta-\varphi^{2} / 2\right) .
$$

By (2.25) and (2.32) we have

$$
0 \leq u(x) \leq \hat{u} \text { in } \bar{\Omega} .
$$

We are now in a position to state the main result of this section.

THEOREM 2.1. There exists at least a regular solution of problem $\mathrm{Pb}_{1}$.

Proof. Let $A=\left\{w(x) \in C^{0}(\bar{\Omega}), 0 \leq w(x) \leq \hat{u}\right\}$. Fix $w(x) \in A$ and consider the linear problem

$$
\begin{gathered}
\nabla \bullet(\sigma(w) \nabla \varphi)=0 \quad \text { in } \Omega, \\
\varphi=0 \quad \text { on } S_{0}, \quad \frac{d \varphi}{d n}=0 \quad \text { on } S_{2}, \\
\varphi+R \int_{S_{1}} \sigma(w) \frac{d \varphi}{d n} d s=V \quad \text { on } S_{1} .
\end{gathered}
$$


By Lemma 2.1 there exists one and only one solution $\varphi \in C^{0, \alpha}(\bar{\Omega}), \alpha \in(0,1)$, of problem (2.35)-(2.37). Besides, we have $0 \leq \varphi \leq V$ in $\bar{\Omega}$ and, by standard results of regularity,

$$
\|\varphi\|_{C^{0 . \alpha}(\bar{\Omega})} \leq C_{1}
$$

where the constant $C_{1}$ depends only on $\sigma_{0}, \sigma_{1}, V$, and $\Omega$. Next we solve the problem

$$
\begin{gathered}
\nabla \bullet(\sigma(w) \nabla \theta)=0 \quad \text { in } \Omega, \\
\theta=F\left(u_{0}\right) \quad \text { on } S_{0}, \quad \theta=F\left(u_{0}\right)+\frac{\varphi_{1}^{2}}{2} \quad \text { on } S_{1}, \quad \frac{d \theta}{d n}=0 \quad \text { on } S_{2},
\end{gathered}
$$

where $\varphi_{1}$ is the constant value on $S_{1}$ of the solution $\varphi(x)$ of $(2.35)-(2.37)$. As in (2.32) we have $0 \leq \theta \leq \theta_{M}$ in $\bar{\Omega}$ and $\theta \in C^{0, \alpha}(\bar{\Omega})$. Moreover, by elliptic estimates, we obtain

$$
\|\varphi\|_{C^{0, \beta}(\bar{\Omega})} \leq C_{2}, \quad \beta \in(0,1),
$$

where the constant $C_{2}$ depends only on the data and not on $w$. Define

$$
u(x)=F^{-1}\left(\varphi^{2}(x) / 2-\theta(x)\right) .
$$

We get $0 \leq u \leq \hat{u}$ in $\bar{\Omega}$ and

$$
\|u\|_{C^{0, \gamma}(\bar{\Omega})} \leq C_{3}, \quad \gamma \in(0,1) .
$$

It follows that the mapping $u=T w$ maps $A$ into a compact subset of $A$. It is also easy to check that $T$ is continuous. Hence by the fixed point theorem of $\mathrm{J}$. Schauder, $T$ has a fixed point. By the usual bootstrap argument, we conclude that the regularity of the solution of problem $\mathrm{Pb}_{1}$ depends only on the assumptions of regularity made on $\sigma, K$, and $\partial \Omega$.

REMARK 2.1. Uniqueness is not to be expected for problem $\mathrm{Pb}_{1}$ without further hypotheses on $\sigma$ and $\kappa$. An example of nonuniqueness is given in [1].

3. Heating in massive conductor caused by the eddy currents. In certain industrial processes small pieces of metal are brought to the melting point with the intense Jouleheating generated by the Foucault currents induced in the specimen by an external variable magnetic field. Now the Wiedemann-Franz law relates the electrical and thermal conductivities with the temperature in metals and reads $\sigma=C k / u$, where $C$ is a universal constant. This implies a substantial variation for $\sigma$ in the range of temperature practically encountered. In this section we propose a model, valid for a geometrically simple situation, for describing the heating due to the eddy currents. A theorem of existence of solution is also given.

Let us consider a long, conducting, and homogeneous cylinder of magnetic permeability $\mu$ and orthogonal cross-section $\Omega$, an open and bounded subset of $\mathbb{R}^{2}$ with a regular boundary $\partial \Omega$. An insulating medium of permeability $\mu_{0}$ fills the space utside the cylinder. Moreover, a time-periodic magnetic field is given at infinity by

$$
\mathbf{H}_{\infty}=\bar{H}(\tau) \mathbf{i}_{3},
$$


where $\bar{H}(\tau)$ is a $T$-periodic regular function and $\mathbf{i}_{3}$ is the unit vector parallel to the axis of the cylinder. We want to find the magnetic field $\mathbf{H}$ everywhere, the current density $\mathbf{J}$ and the temperature $U$ in the cylinder corresponding to the Joule heating due to the eddy currents. Given the geometry of the situation, we assume

$$
\mathbf{H}=H(X, \tau) \mathbf{i}_{3}, \quad X=\left(X_{1}, X_{2}\right) .
$$

By (3.2) the equation

$$
\nabla \bullet \mathbf{H}=0
$$

is satisfied.

We assume we operate with quasi-stationary fields, thus we neglect, the displacement's current in the Maxwell equation

$$
\nabla \times \mathbf{H}=\mathbf{J}+\frac{1}{C^{2}} \frac{\partial \mathbf{E}}{\partial t} .
$$

Outside the cylinder, where $\mathbf{J}=0$, we have

$$
\nabla \times \mathbf{H}=0,
$$

i.e., by (3.2), $\nabla H=0$. This implies the constancy in space of $\mathbf{H}$ in the insulating medium. Inside the cylinder we get, by Ohm's law and (3.4),

$$
\nabla \times \mathbf{H}=\mathbf{J}=\sigma \mathbf{E} .
$$

Hence

$$
\mathbf{E}=\rho \nabla H \times \mathbf{i}_{3},
$$

where $\rho=1 / \sigma$. Recalling that

$$
\nabla \times \mathbf{E}=-\mu \mathbf{H}_{\tau}
$$

we obtain from (3.7)

$$
\nabla \bullet(\rho \nabla H)=\mu H_{\tau} .
$$

The energy equation reads in the present case

$$
\varepsilon c U_{\tau}-\nabla \cdot(\kappa \nabla U)=\rho|\nabla H|^{2},
$$

since the expression for the Joule heating is $\mathbf{E} \bullet \mathbf{J}=\rho|\nabla H|^{2} . \operatorname{In}(3.10) \varepsilon$ is the mass density, $c$ the specific heat, and $\kappa$ the thermal conductivity. By the continuity of $H$ across the surface of the cylinder we obtain

$$
H=\bar{H}(\tau) \quad \text { on } \partial \Omega \times[0, \infty) .
$$

In addition, we assume

$$
\begin{gathered}
U(X, 0)=0, \\
U=0 \quad \text { on } \partial \Omega \times[0, \infty) .
\end{gathered}
$$

The thermal and electrical conductivities are supposed to be given functions of th temperature. 
If $L, H_{0}, U_{0}, \rho_{0}$, and $\kappa_{0}$ are respectively characteristic constants for length, magnetic field, temperature, resistivity, and thermal conductivity, we can write the basic equations in nondimensional form defining

$$
\begin{aligned}
& x=X / L, \quad t=\tau / T, \quad h(x, t)=H(L x, T t) / H_{0}, \\
& \theta(x, t)=U(L x, T t) / U_{0}, \quad r=\rho / \rho_{0}, \quad k=\kappa / \kappa_{0} .
\end{aligned}
$$

Equations (3.9) and (3.10) become

$$
\begin{gathered}
a_{1} h_{t}-\nabla \bullet(r(\theta) \nabla h)=0, \\
a_{2} \theta_{t}-\nabla \cdot(k(\theta) \nabla \theta)=a_{3} r(\theta)|\nabla h|^{2},
\end{gathered}
$$

where $a_{1}, a_{2}$, and $a_{3}$ are nondimensional parameters given by

$$
a_{1}=\frac{\mu L^{2}}{\rho_{0} T}, \quad a_{2}=\frac{\varepsilon c L^{2}}{\kappa_{0} T}, \quad a_{3}=\frac{\rho_{0} H_{0}^{2}}{U_{0} \kappa_{0}} .
$$

We want to treat the case in which the ratio $a_{2} / a_{1}$ is much smaller than 1 . This is not an unrealistic assumption for certain highly ferromagnetic substances like Si-Fe crystals for which $a_{2} / a_{1}$, as computed from the data for $\mu$ given in [4, p. 374], is of order $10^{-3}$. For this reason we neglect, in a rather heuristic way, the time derivative in (3.15). This implies of course the impossibility of satisfying the initial condition $\theta(x, 0)=0$. In this way we study the quasi-stationary situation which occurs after the body is fully heated up. Let us assume

$$
\begin{aligned}
& r_{1} \geq r(\theta) \geq r_{0}>0, \\
& k_{1} \geq k(\theta) \geq k_{0}>0 .
\end{aligned}
$$

Putting

$$
F(\theta)=\int_{0}^{\theta} k(\zeta) d \zeta
$$

we can define a new scale of temperature $u=F(\theta)$. By (3.18), $F$ maps $[0, \infty)$ on $[0, \infty)$ one-to-one. Let $d(u)=r\left[F^{-1}(u)\right]$. We obtain the following problem:

$$
\begin{gathered}
h_{t}-\nabla \bullet(d(u) \nabla h)=0, \\
h=h_{0}(t) \quad \text { on } \partial \Omega \times[0, \infty), \\
h(x, t)=h(x, t+1), \\
-\Delta u=d(u)|\nabla h|^{2}, \\
u=0 \quad \text { on } \partial \Omega \times[0, \infty),
\end{gathered}
$$

where we assume $h_{0}(t)=h_{0}(t+1)$. We discuss first of all the nonphysical, but elementary case in which

$$
h_{0}(t)=e^{-i 2 \pi t}
$$


We look for solutions of problem (3.20)-(3.25) of the form $h(x, t)=$ $e^{-i 2 \pi t}[v(x)+i w(x)]$. Substituting in (3.20)-(3.23) we arrive at the following nonlinear elliptic system:

$$
\begin{array}{cc}
\nabla \bullet(d(u) \nabla v)-w=0, & v=1 \text { on } \partial \Omega, \\
\nabla \bullet(d(u) \nabla w)+v=0, & w=0 \text { on } \partial \Omega, \\
-\Delta u=d(u)\left[|\nabla v|^{2}+|\nabla w|^{2}\right], & u=0 \text { on } \partial \Omega .
\end{array}
$$

In view of (3.26), (3.27), and (3.28) we obtain, with a simple calculation,

$$
\nabla \cdot[d(u) \nabla \gamma]=0,
$$

where

$$
\gamma=\frac{v^{2}}{2}+\frac{w^{2}}{2}+G(u), \quad G(u)=\int_{0}^{u} \frac{d t}{d(t)} .
$$

Since $\gamma=\frac{1}{2}$ on $\partial \Omega$ and $d_{1} \geq d(u) \geq d_{0}>0$, we conclude

$$
\frac{v^{2}}{2}+\frac{w^{2}}{2}+G(u)=\frac{1}{2} \text {. }
$$

On the other hand, by the maximum principle we have $u \geq 0$ in $\bar{\Omega}$. Hence $|v|$, $|w|$, and $u$ are pointwise bounded by an absolute constant. Moreover, since $G$ maps $[0, \infty)$ on $[0, \infty)$ one-to-one, we can write

$$
u=G^{-1}\left[\frac{1}{2}-\frac{v^{2}}{2}-\frac{w^{2}}{2}\right]
$$

and problem (3.26)-(3.28) can be restated as follows:

$$
\begin{array}{ll}
\nabla \bullet[A(v, w) \nabla v]-w=0, & v=1 \text { on } \partial \Omega, \\
\nabla \bullet[A(v, w) \nabla w]+v=0, & w=0 \text { on } \partial \Omega,
\end{array}
$$

where $A(v, w)=d\left[G^{-1}\left(\frac{1}{2}-v^{2} / 2-w^{2} / 2\right)\right]$.

The Schauder's fixed point theorem can be applied quite easily to problem (3.31)(3.32) and the existence of at least one classical solution can in this way be proved.

4. Existence for the eddy currents problem. In this section we study the following problem $\mathrm{Pb}_{2}$ :

$$
\begin{gathered}
h_{t}-\nabla \bullet(d(u) \nabla h)=0, \\
h=h_{0}(t) \quad \text { on } \partial \Omega \times[0, \infty), \\
h(x, t)=h(x, t+1),
\end{gathered}
$$

where $h_{0}(t)$ is now an arbitrary 1-periodic, regular function. Integrating equation (3.23) over one time period we obtain

$$
-\Delta u=d(u) \int_{0}^{1}|\nabla h|^{2} d t, \quad u=0 \text { on } \partial \Omega .
$$

We want to prove that problem (3.20), (3.21), (3.22), (4.1) has at least one weak solution. Let $Q=\Omega \times(0,1), N=\Omega \times \mathbb{R}^{1}$, and $S=\partial \Omega \times \mathbb{R}^{1}$. Let $C_{0}^{\infty}(Q)$ be 
the set of all functions $\eta(x, t)$ of class $C^{\infty}(\bar{N})$, periodic in $t$ with period 1 and vanishing near $S$. Define $\stackrel{\circ}{W}_{2}^{1.0}(Q)$ as the closure of $C_{0}^{\infty}(Q)$ with respect to the norm

$$
\|\eta\|_{W_{2}^{1,0}(Q)}=\left[\int_{Q} v^{2} d x d t+\int_{Q}|\nabla v|^{2} d x d t\right]^{1 / 2},
$$

where $\nabla \eta=\left(\eta_{x_{1}}, \eta_{x_{2}}\right)$. Let $\stackrel{\circ}{W}_{2}^{1,1}(Q)$ be the closure of $C_{0}^{\infty}(Q)$ with respect to the norm

$$
\|\eta\|_{\dot{W}_{2}^{1,1}(Q)}=\left[\int_{Q} v^{2} d x d t+\int_{Q}|\nabla v|^{2} d x d t+\int_{Q} v_{t}^{2} d x d t\right]^{1 / 2} .
$$

As a weak formulation of problem $\mathrm{Pb}_{2}$ we take

$$
h-h_{0} \in \stackrel{\circ}{W}_{2}^{1,0}(Q), \quad \int_{Q} h \eta_{t} d x d t-\int_{Q} d(u) \nabla h \bullet \nabla \eta d x d t=0
$$

for all $\eta \in \stackrel{\circ}{W}_{2}^{1,1}(Q)$, and

$$
u \in H_{0}^{1}(\Omega), \quad \int_{\Omega} \nabla u \bullet \nabla v d x=\int_{Q} d(u)|\nabla h|^{2} v d x d t
$$

for all $v \in \stackrel{\circ}{H}^{1}(\Omega) \cap L^{\infty}(\Omega)$, where $\stackrel{\circ}{H}^{1}(\Omega)$ is the usual Sobolev space obtained as completion of the function $v(x) \in C^{\infty}(\bar{\Omega})$ vanishing near $\partial \Omega$ with respect to the norm

$$
\|v\|_{H^{1}(\Omega)}=\left[\int_{\Omega}|\nabla v|^{2} d x\right]^{1 / 2} .
$$

The main difficulty in treating problem $\mathrm{Pb}_{2}$ lies in the fact that the left-hand side of Eq. (4.1) belongs "a priori" only to $L^{1}(\Omega)$. For this reason we consider the following sequence of approximating problems $\mathrm{Pb}_{n}$ :

$$
\begin{gathered}
\frac{\partial h_{n}}{\partial t}-\nabla \bullet\left(d\left(u_{n}\right) \nabla h_{n}\right)=0, \\
h_{n}=h_{0} \quad \text { on } S, \quad h_{n}(x, t)=h_{n}(x, t+1), \\
\frac{1}{n} \Delta \Delta u_{n}-\Delta u_{n}=d\left(u_{n}\right) \int_{0}^{1}\left|\nabla h_{n}\right|^{2} d t, \\
u_{n}=0, \quad \Delta u_{n}=0 \quad \text { on } \partial \Omega,
\end{gathered}
$$

and the corresponding weak formulation: $h_{n}-h_{0} \in \stackrel{\circ}{W}_{2}^{1,0}(Q)$,

$$
\int_{Q} h_{n} \eta_{t} d x d t-\int_{Q} d\left(u_{n}\right) \nabla h_{n} \bullet \nabla \eta d x d t=0
$$

for all $\eta \in \stackrel{\circ}{W}_{2}^{1,1}(Q)$,

$$
\begin{aligned}
& u_{n} \in \stackrel{\circ}{H}^{1}(\Omega) \cap H^{2}(\Omega), \\
& \frac{1}{n} \int_{\Omega} \Delta u_{n} \Delta v d x+\int_{\Omega} \nabla u_{n} \cdot \nabla v d x=\int_{Q} d\left(u_{n}\right)\left|\nabla h_{n}\right|^{2} v d x d t,
\end{aligned}
$$

for all $v \in \stackrel{\circ}{H}^{1}(\Omega) \cap H^{2}(\Omega)$. 
First of all we derive various "a priori" estimates for the solutions of problem $\mathrm{Pb}_{n}$. By the parabolic maximum principle we have in $Q$ :

$$
\min _{[0,1]} h_{0}(t) \leq h_{n}(x, t) \leq \max _{[0,1]} h_{0}(t) .
$$

Moreover if we proceed as in Lemma 3.1 of [2], where a similar situation occurs, we can show that

$$
u_{n} \geq 0 \text { in } \bar{\Omega} \text {. }
$$

Since $u_{n} \in H^{2}(\Omega)$, we have $h_{n}-h_{0} \in \stackrel{\circ}{W}_{2}^{1,1}(Q)$. Choosing $\eta=h_{n}-h$ in (4.8) we obtain

$$
\int_{Q} d\left(u_{n}\right)\left|\nabla h_{n}\right|^{2} d x d t \leq C_{1}
$$

and, by (3.17),

$$
\int_{Q}\left|\nabla h_{n}\right|^{2} d x d t \leq C_{2}
$$

where the constants $C_{1}$ and $C_{2}$ do not depend on $n$. Let us multiply (4.4) by $h_{n} u_{n}$. Since $h_{n} u_{n}=0$ on $S$, integrating by part over $\Omega$ we have

$$
\frac{1}{2} \frac{d}{d t} \int_{\Omega} h_{n}^{2} u_{n} d x+\int_{\Omega} u_{n} d\left(u_{n}\right)\left|\nabla h_{n}\right|^{2} d x+\int_{\Omega} h_{n} d\left(u_{n}\right) \nabla h_{n} \cdot \nabla u_{n} d x=0 .
$$

By (4.10) and (4.12) we infer

$$
\int_{Q} u_{n} d\left(u_{n}\right)\left|\nabla h_{n}\right|^{2} d x d t \leq C_{3}\left[\int_{Q}\left|\nabla u_{n}\right|^{2} d x d t\right]^{1 / 2} .
$$

Selecting $v=u_{n}$ in (4.9), we have by (4.13)

$$
\frac{1}{n} \int_{\Omega}\left|\Delta u_{n}\right|^{2} d x+\int_{\Omega}\left|\nabla u_{n}\right|^{2} d x \leq C_{3}\left[\int_{\Omega}\left|\nabla u_{n}\right|^{2} d x\right]^{1 / 2} .
$$

It follows that

$$
\begin{gathered}
\int_{\Omega}\left|\nabla u_{n}\right|^{2} d x \leq C, \\
\frac{1}{n} \int_{\Omega}\left|\Delta u_{n}\right|^{2} d x \leq C,
\end{gathered}
$$

where again $C$ is a constant not depending on $n$.

The following lemma is a straightforward application of the Schauder fixed point theorem.

LemMa 4.1. For every fixed $n \in \mathbb{N}$, there exists at least one solution of problem $\mathrm{Pb}_{n}$.

Sketch of Proof. We omit the index $n$. Let $w \in \stackrel{\circ}{H}^{1}(\Omega)$ and solve, using standard results of linear theory, the problem

$$
h-h_{0} \in \stackrel{\circ}{W}_{2}^{1,0}(Q), \quad \int_{Q} h \eta_{t} d x d t-\int_{Q} d(w) \nabla h \cdot \nabla \eta d x d t=0
$$


for all $\eta \in \stackrel{\circ}{W}_{2}^{1,1}(Q)$. Then we solve

$$
\begin{aligned}
& u \in \stackrel{\circ}{H}^{1}(\Omega) \cap H^{2}(\Omega), \\
& \int_{\Omega} \Delta u \Delta v d x+\int_{\Omega} \nabla u \cdot \nabla v d x=\int_{Q} d(u)|\nabla h|^{2} v d x d t
\end{aligned}
$$

for all $v \in \stackrel{\circ}{H}^{1}(\Omega) \cap H^{2}(\Omega)$.

By the estimates (4.12), (4.14), and (4.15) there exists $R>0$ such that the map $u=T w$ maps the ball $B_{R}$ of center zero and radius $R$ of $\stackrel{\circ}{H}^{1}(\Omega)$ into itself. By (4.15) the set $T\left(B_{R}\right)$ is compact in $\stackrel{\circ}{H}^{1}(\Omega)$. Besides, $T$ is continuous, hence $T$ has a fixed point and problem $\mathrm{Pb}_{n}$ a solution.

By (4.10), (4.12), and (4.14) we can extract from $\left\{h_{n}, u_{n}\right\}$ a subsequence $\left\{h_{m}, u_{m}\right\}$ such that

$$
\begin{aligned}
h_{m} & \rightarrow h & & \text { strongly in } L^{2}(Q), \\
\nabla h_{m} & \rightarrow \nabla h & & \text { weakly in } L^{2}(Q), \\
u_{m} & \rightarrow u & & \text { strongly in } L^{2}(Q), \\
\nabla u_{m} & \rightarrow \nabla u & & \text { weakly in } L^{2}(Q), \\
d\left(u_{m}\right) & \rightarrow d(u) & & \text { strongly in } L^{p}(Q), \quad 1 \leq p<\infty, \\
d\left(u_{m}\right) & \rightarrow d(u) & & \text { a.e. in } \Omega .
\end{aligned}
$$

By (4.16), (4.17), and (4.20) we can pass to the limit in (4.8). We get

$$
h-h_{0} \in \stackrel{\circ}{W}_{2}^{1,0}(Q), \quad \int_{Q} h \eta_{t} d x d t-\int_{Q} d(u) \nabla h \cdot \nabla \eta d x d t=0
$$

for all $\eta \in \stackrel{\circ}{W}_{2}^{1,1}(Q)$.

From (4.8) and (4.22) we obtain

$$
\begin{gathered}
\int_{Q} d\left(u_{m}\right) \nabla\left(h_{m}-h\right) \bullet \nabla \eta d x d t-\int_{Q}\left(h_{m}-h\right) \eta_{t} d x d t \\
=\int_{Q}\left(d(u)-d\left(u_{m}\right)\right) \nabla h \bullet \nabla \eta d x d t,
\end{gathered}
$$

for all $\eta \in \stackrel{\circ}{W}_{2}^{1,1}(Q)$.

By (4.20) and (4.23) we conclude that

$$
\begin{gathered}
\int_{Q} d\left(u_{m}\right)\left|\nabla\left(h_{m}-h\right)\right|^{2} d x d t \rightarrow 0, \\
\nabla h_{m} \rightarrow \nabla h \text { strongly in } L^{2}(Q) .
\end{gathered}
$$

On the other hand,

$$
\begin{aligned}
d\left(u_{m}\right)\left|\nabla h_{m}\right|^{2}= & d\left(u_{m}\right)\left|\nabla\left(h_{m}-h\right)\right|^{2} \\
& +2 d\left(u_{m}\right) \nabla h \bullet \nabla h_{m}-d\left(u_{m}\right)|\nabla h|^{2},
\end{aligned}
$$


hence we obtain, by (4.24), (4.25), and (4.20),

$$
\int_{Q} d\left(u_{m}\right)\left|\nabla h_{m}\right|^{2} d x d t \rightarrow \int_{Q} d(u)|\nabla h|^{2} d x d t .
$$

From (4.20), (4.25), and (4.26) we have, using Lebesgue's dominated convergence theorem,

$$
d\left(u_{m}\right)\left|\nabla h_{m}\right|^{2} \rightarrow d(u)|\nabla h|^{2} \quad \text { strongly in } L^{1}(Q) .
$$

We are now in a position to pass to the limit in (4.9) as $m \rightarrow \infty$. By (4.15) we infer

$$
\frac{1}{m} \int_{\Omega} \Delta u_{m} \Delta v d x \rightarrow 0
$$

and by (4.19)

$$
\int_{\Omega} \nabla u_{m} \bullet \nabla v d x \rightarrow \int_{\Omega} \nabla u \bullet \nabla v d x
$$

Recalling (4.27) we obtain

$$
\int_{Q} d\left(u_{m}\right)\left|\nabla h_{m}\right|^{2} v d x d t \rightarrow \int_{Q} d(u)|\nabla h|^{2} v d x d t
$$

Whence we have, for all $v \in \stackrel{\circ}{H}^{1}(\Omega) \cap H^{2}(\Omega)$,

$$
u \in \stackrel{\circ}{H}^{1}(\Omega), \quad \int_{\Omega} \nabla u \bullet \nabla v d x=\int_{Q} d(u)|\nabla h|^{2} d x d t .
$$

By density, (4.28) holds true also for all $v \in \stackrel{\circ}{H}^{1}(\Omega) \cap L^{\infty}(\Omega)$. Hence (4.3) follows. This proves that problem $\mathrm{Pb}_{2}$ has at least one weak solution.

\section{REFERENCES}

[1] G. Cimatti, The stationary thermistor problem with a current limiting device, Proc. Roy. Soc. Edinburgh Sect. 116A, 79-84 (1990)

[2] G. Cimatti and G. Prodi, Existence results for a nonlinear elliptic system modelling a temperature dependent electrical resistor, Ann. Mat. Pura Appl. 62, 227-236 (1988)

[3] A. C. Fowler, S. D. Howison, and E. J. Hinch, Temperature surges in current-limiting circuit devices, to appear

[4] H. A. Haus and J. R. Melcher, Electromagnetic Fields and Energy, Prentice-Hall, Englewood Cliffs, $\mathrm{NJ}, 1989$

[5] O. A. Ladyzenskaja, V. A. Solonnikov, and N. N. Ural'ceva, Linear and quasilinear equations of parabolic type, Transl. Math. Monographs, vol. 23, Amer. Math. Soc., Providence, RI, 1968

[6] J. L. Lions, Equations Différentielles Operationelles et Problémes aux Limites, Springer-Verlag, Berlin, 1961

[7] M. H. Protter and H. F. Weinberger, Maximum Principle in Partial Differential Equations, Prentice-Hall, Englewood Cliffs, NJ, 1967 